\title{
Model for non-perturbative sea quark and gluon generation
}

\author{
H. R. Christiansen* \\ Group of Theoretical Physics, Universidade Católica de Petrópolis, and Centro Brasileiro de \\ Pesquisas Físicas CBPF-DCP, Rua Dr. Xavier Sigaud 150, 22290-180, Rio de Janeiro, Brazil \\ E-mail: hhugo@cbpf. bri
}

ABstract: We propose a model description for the low $Q^{2}$ parton distribution functions in nucleons which allows an accurate fit of the recently measured light sea $S U(2)$ symmetry breaking with just two parameters. Furthermore, a novel mechanism for generating the primordial gluon density which is needed to fit experimental data on parton distributions is put forward. Our model is based on an appropriate interplay between hadronic fluctuations and quark degrees of freedom at the valon scale.

\section{Introduction}

The structure of hadrons in the framework of Quantum Chromodynamics can be suitably analysed by means of parton distribution functions in the infinite momentum frame. As is wellknown, the foregoing can be obtained from experimental cross sections in hadron-hadron and lepton-hadron collisions. Hence, the theoretical control of such distributions is a crucial issue for the understanding of the fundamentals of particle physics.

The internal dynamics of a hadron may then be investigated when one considers it in terms of parton distribution functions. Thus, the structure of a single hadron can be directly connected to the experimental data from scattering processes and, out of these data, improve our knowledge of the dynamics of the microscopic world underlying each kind of hadron.

Parton distribution functions are interpreted as the probability density of finding a parton (quark or gluon) with a fraction of the hadron momentum when probed by some momentum transfer $Q^{2}$. The $Q^{2}$ dependence of parton densities is well described by the DGLAP [i] evolution equations within perturbative QCD. However, in

*Collaborator: J. Magnin, Universidad de los Andes, AA4976 Bogotá, Colombia. order to have adequate fits to the Deep Inelastic Scattering (DIS) data, initial (valence-like) distributions at a low resolution scale must be given as an input. Although repeatedly addressed in

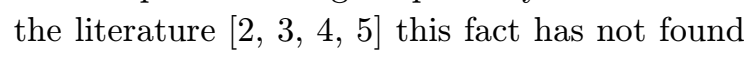
a satisfactory explanation and the origin of such initial distributions is still unclear.

A plan for unveiling the primordial low scale parton densities should be traced back to the internal dynamics of the corresponding hadronic bound state. Put in other words, low scale parton distributions depend on the confining properties of QCD, which are expected to give the ultimate response to the origin of hadrons as bound states of quarks and gluons. It is worth noting that sea quark and gluon distributions at a low resolution scale can be related to the old intrinsic sea idea. Following [6], intrinsic quarks and gluons exist over a time scale independent of any probe momentum and are associated with the bound-state hadron dynamics. On the other hand, the socalled extrinsic sea of gluons and quarks have a purely perturbative origin and their distributions show the characteristics of bremsstrahlung and pair production processes leading to the standard DGLAP perturbative QCD evolution.

These considerations led us to draw a low $Q^{2}$ picture of hadrons [iㄱㅁ] in terms of effective quark degrees of freedom, interacting among them thr- 
ough sea quarks and gluons. The building blocks are the so-called valons which are valence quarks appropriatedly dressed by their extrinsic sea Within our picture one can represent the hadron wave function as a superposition of hadron-like Fock states which we construct by means of a well-known recombination mechanism [9in. For example, the proton wave function can be written as

$$
|p\rangle=a_{0}\left|p_{\circ}\right\rangle+a_{1}\left|\hat{p}_{\circ} g\right\rangle+\sum_{i} a_{i}\left|B_{i} M_{i}\right\rangle
$$

at some low $Q_{v}^{2}$ scale compatible with the valon picture of the proton. Here $\left|p_{\circ}\right\rangle$ is a pure three

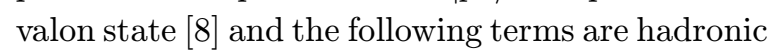
quantum fluctuations which emerge from the (nonperturbative) interaction among the valons. In particular, the second term includes the leading non-perturbative gluon distribution which is dinamically generated in our approach. The hat is for distinguishing possible differences between the bare proton in the two different configurations.

Restricting the series to the first term corresponds to the resolution of the old fashioned fits to experimental DIS data, which resolve just the structure of the valons themselves. This makes the following terms crucial to provide a realistic description of the proton. Through convolution formulae, these terms give rise to the initial nonperturbative sea densities generally taken as an input in phenomenological fits. Therefore, their role is to provide the low $Q^{2}$ parton distributions in the hadron wave-function. In a sense, they give the nonperturbative binding between the constituent quarks (valons), necessary to build the physical hadron.

This representation of the proton wave function led in a natural way to a $\bar{d} / \bar{u}$ asymmetry in the proton sea which closely describes [1] $\left.{ }^{1}\right]$ the most recent experimental data by the E866 Collaboration (see Fig. in Ref. $\overline{\overline{7}} \overline{1}]$ a $s-\bar{s}$ asymmetry in the nucleon sea was calculated, qualitatively agreeing with the results of the last global analysis of DIS data [1 [12

In the following section, we determine the low $Q^{2}$ non-perturbative gluon distributions in nucleons, using the model introduced in Ref. $\overline{\nabla 7}$. Finally, section ${ }_{14}^{4}$ is devoted to the conclusions.
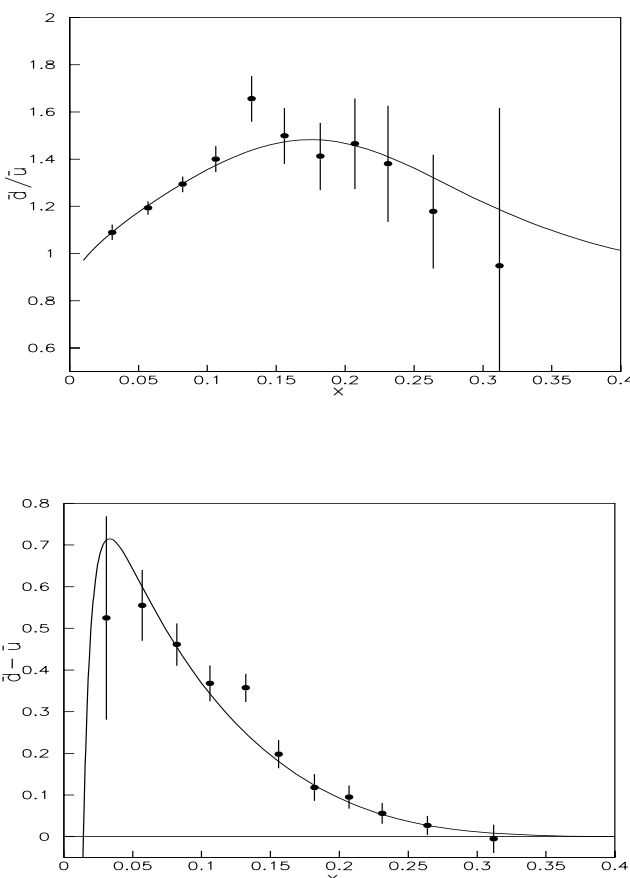

Figure 1: Model predictions for $\bar{d} / \bar{u}$ (upper) and $\bar{d}-\bar{u}$ (lower) distributions in the proton evolved up to $Q^{2}=54.02 \mathrm{GeV}^{2}$. Experimental data are taken from Ref. [1] 1]

\section{The model}

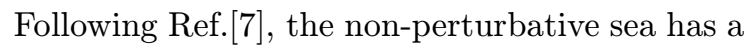
two-step origin in our model. In the first step a valon emits a gluon which subsequently decays into a quark-antiquark pair. In the second, such quark and antiquark interact with the valons and give rise to a bound $|M B\rangle$ state. The in mesonbaryon parton densities give ultimately the nonperturbative quark and antiquark proton distributions.

The emission of a gluon out of a valon is a basic QCD process which can be adequately described in terms of the convolution of the initial valon distribution, $v(z)$, with the Altarelli-Parisi $P_{g q}(z)$ and $P_{q g}(z)$ splitting functions [i1]. In this way, the quark and antiquark initial distributions are given by $[\overline{7}]$

$$
\begin{aligned}
q(x)= & \bar{q}(x)=N \frac{\alpha_{s t}^{2}\left(Q_{v}^{2}\right)}{(2 \pi)^{2}} \int_{x}^{1} \frac{d y}{y} \\
& P_{q g}\left(\frac{x}{y}\right) \int_{y}^{1} \frac{d z}{z} P_{g q}\left(\frac{y}{z}\right) v(z) .
\end{aligned}
$$


Notice that as the valon distribution does not depend on $Q$, the scale dependence in eq. (2.1i) only arises through the strong coupling constant $\alpha_{s t}$. However, it is fixed by the valon model scale, namely $Q^{2}=0.64 \mathrm{GeV}^{2}$. Consequently, pair creation can be safely evaluated in a perturbative way since $\left(\alpha_{s t} / 2 \pi\right)^{2}$ is still sufficiently small. Now, provided such sea $q$ and $\bar{q}$ live sufficiently long, they have a probability to recombine with the remaining valons. Once recombination is accomplished, it can give rise to a (virtual) hadronic bound state. Since such a situation is in the realm of confinement, non-perturbative strong interactions are implicitly understood in recombination. Therefore, the rearrangement of such five component configuration into a meson - baryon bound state must be evaluated by means of effective methods. Assuming that the in-nucleon meson and baryon formation arises from mechanisms similar to those at work in the production of real hadrons, we can evaluate the in-proton meson probability density using the Das-Hwa recombination approach [īi].

Thus, in our model, the in-nucleon meson distributions are given by

$$
P_{M_{i} B_{i}}(x)=\int_{0}^{1} \frac{d y}{y} \int_{0}^{1} \frac{d z}{z} F(y, z) R(x, y, z),
$$

where

$$
F(y, z)=\beta y v(y) z \bar{q}(z)(1-y-z)^{a},
$$

is the valon-antiquark distribution and $R(x, y, z)$ $=\alpha \frac{y z}{x^{2}} \delta\left(1-\frac{y+z}{x}\right)$ is the recombination function $(x, y$ and $z$ are the momentum fractions of the in-nucleon meson, the valon and the antiquark respectively).

Due to momentum conservation, the in- nucleon meson and baryon probability densities are not independent but correlated by

$$
P_{M_{i} B_{i}}(x)=P_{B_{i} M_{i}}(1-x),
$$

with an additional correlation in velocity given by

$$
\frac{\left\langle x P_{M_{i} B_{i}}(x)\right\rangle}{m_{M_{i}}}=\frac{\left\langle x P_{B_{i} M_{i}}(x)\right\rangle}{m_{B_{i}}} .
$$

The above constraint $(2.5 i)$, which is needed in order to build a $\left|M_{i} B_{i}\right\rangle$ bound state, fixes the exponent $a$ in eq. $\left(2.3_{1}^{i}\right)\left[10_{1}^{\prime \prime}\right.$.
The hadronic fluctuations so far described can be interpreted as the origin of the intrinsic quark-antiquark sea. As a consequence, since the resulting $\bar{q}$ and $q$ sea distributions belong to different terms or components in the Fock series, intrinsic quark and antiquark probability densities are unequal in a general way.

At this point, one has to decide what fluctuations should be included in the expansion (11.i. For definitness, consider the proton wave function. Taking into account mass values and quantum numbers, the main fluctuations of the proton should be the $\left|\pi^{+} n\right\rangle,\left|\pi^{+} \Delta^{0}\right\rangle$ and $\left|\pi^{-} \Delta^{++}\right\rangle$ virtual states, with probabilities $a_{\pi n}^{2}$ and $a_{\pi \Delta}^{2}$ respectively. Differences between the $\left|\pi^{+} \Delta^{0}\right\rangle$ and $\left|\pi^{-} \Delta^{++}\right\rangle$probabilities are given by ClebshGordan coefficients which ensure the correct global isospin of the fluctuation. Thus, we obtain $\frac{1}{6} a_{\pi \Delta}^{2}$ and $\frac{1}{2} a_{\pi \Delta}^{2}$ for $\left|\pi^{+} \Delta^{0}\right\rangle$ and $\left|\pi^{-} \Delta^{++}\right\rangle$respectively. On the other hand, the probability of the $\left|\pi^{+} n\right\rangle$ bound-state is $\frac{2}{3} a_{\pi n}^{2}$. The coefficients $a_{\pi n}^{2}$ and $a_{\pi \Delta}^{2}$ are given by $(N \alpha \beta)_{\pi n}$ and $(N \alpha \beta)_{\pi \Delta}$ respectively. Their numerical values result from comparison with experimental data. Fluctuations like $|\rho N\rangle,|\rho \Delta\rangle$, etc. which could contribute, for instance, to the $\bar{d}-\bar{u}$ asymmetry in the proton, are far off-shell ${ }^{1}$ and can be safely neglected.

Remarkably, as shown in Ref. [1] 10$]$, this approach leads to a $\bar{d} / \bar{u}$ asymmetry in the proton which closely describes the experimental data by the E866 Collaboration [i 1in. It is also worth noting that this is, at present, the only available model which can describe equally well the difference $\bar{d}-\bar{u}$ and the ratio $\bar{d} / \bar{u}$ as given by the E866 Collaboration (see Fig. $\left.\underline{1}_{1}^{\prime \prime}\right)$. Including fluctuations to Kaon-Hyperon states, $|K H\rangle$, a $s-\bar{s}$ asymmetry in the proton sea arises [i] which qualitatively agrees with results from a recent global analysis of DIS data

\section{The primordial sea of gluons}

Fluctuations of the proton to $\left|\pi^{0} p\right\rangle$ and $\left|\pi^{0} \Delta\right\rangle$ states do not contribute to the intrinsic quark and antiquark structure. The reason is that the

\footnotetext{
${ }^{1}$ Note that these are even more supressed than strange, $|K H\rangle$, fluctuations.
} 


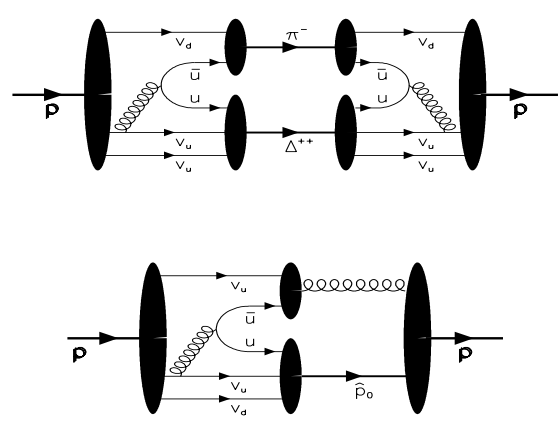

Figure 2: Diagramatic representation of a proton fluctuation (upper). Process leading to the generation of non-perturbative gluons through recombination (lower).

formation of a $\pi^{0}$ in-proton state must be suppressed due to its neutral flavor structure $u \bar{u}-d \bar{d}$. This happens because $v_{q} \bar{q}$ objects can annihilate rapidly into a gluon while $v_{q} \bar{q}^{\prime}$ cannot (here $q^{\prime}$ represents a quark of flavor different to $q$ 's. See Fig. (2,i). Notice also that an unflavored object like a $v_{q} \bar{q}$ pair has itself the quantum numbers of a gluon. Thus, a hypothetical $\left|\pi^{0} p\right\rangle$ does not contribute to the sum over $\left|M_{i} B_{i}\right\rangle$ in the RHS of eq. (1.11) but to the second term, $|p g\rangle$, providing a source of valence-like gluons in the proton. It means that, at leading order, only $\left|\pi^{ \pm} B\right\rangle$ fluctuations are responsible for the intrinsic sea quark and antiquark distributions at the low $Q^{2}$ scale structure of the nucleon.

Concerning time scales, both $\left|\pi^{+} n\right\rangle$ and $|p g\rangle$ fluctuations are expected to last approximately the same. Indeed, the characteristic lifetime of a $|M B\rangle$ fluctuation is proportional to $1 / \Delta E$, where $\Delta E$ is the energy difference between fluctuation and proton states in an infinite momentum frame. Thus, for a $|M B\rangle$ state we have

$$
\tau_{|M B\rangle} \simeq\left[\frac{1}{2 P}\left(\frac{\left(m_{M}\right)_{T}^{2}}{x_{M}}+\frac{\left(m_{B}\right)_{T}^{2}}{x_{B}}-m_{p}^{2}\right)\right]^{-1},
$$

where $P$ is the momentum of the proton in the infinite momentum frame, $m_{M}, m_{B}$ and $m_{p}$ are the masses of meson, baryon and proton and $x_{M}$ and $x_{B}$ are the momentum fractions carried by the meson and the baryon in the fluctuation.
$\left(m_{M, B}\right)_{T}^{2}$ represent the square transverse masses of the virtual hadrons in the fluctuation. Assuming that the neutral objects (the "would be" pions) annihilating into non-perturbative gluons have similar transverse masses we get a similar result for the $|p g\rangle$ lifetime.

Another important point in this approach is that the shape of the intrinsic gluon coming from the $v_{q} \bar{q}$ non-perturbative pairing above described can be also estimated from the probability density of the would be in-proton neutral meson. Since the proposed mechanism involves the mixing of a virtual meson-like object with a gluon via recombination, we can assume that the momentum distribution of the intrinsic gluon is given by the virtual meson distribution. Therefore, we use the recombination formulas for the neutral pion to compute the shape of the intrinsic gluon

$$
\begin{aligned}
g^{N P}\left(x, Q_{v}^{2}\right)= & P_{p g}\left(x, Q_{v}^{2}\right) \equiv N_{p} \frac{(1-x)^{12.9}}{x} \int_{0}^{x} d y \\
& y \bar{q}(y)(y-x) v_{q / p}(y-x), \quad(3.2)
\end{aligned}
$$

where $\bar{q}(x)$ is given by eq. $(2.21 \overline{1}), N_{p}$ is a normalization constant and $v_{N q}(x)$ is the distribution of the $q$-flavored valon in the nucleon given by

$$
v_{N q}(x)=\frac{105}{32} \sqrt{x}(1-x)^{2} .
$$

Here the low scale is fixed by $Q_{v}^{2}=0.64 \mathrm{GeV}^{2}$ as in [8].

An alternative way to have an unflavored meson-Nucleon fluctuation would arise through the self-recombination of the $q-\bar{q}$ pair produced by the gluon splitting of eq. 2 . In this case the unflavored meson should be a vector meson since it was originated by a single gluon. However, this kind of fluctuations consisting of disconnected $M^{0}$ and $N$, are strongly supressed by the OZI rule.

In Fig. 13 we display the intrinsic gluon distribution at the valon scale, given by eq. (3.2), as compared to the initial gluon distribution of the GRV-94 HO [2] parametrization at $Q^{2}=0.4$ $\mathrm{GeV}^{2}$ and the intrinsic gluon distribution calculated in a Monte Carlo based model of the pro-

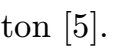

\section{Conclusion}

In this paper we have analysed some important 


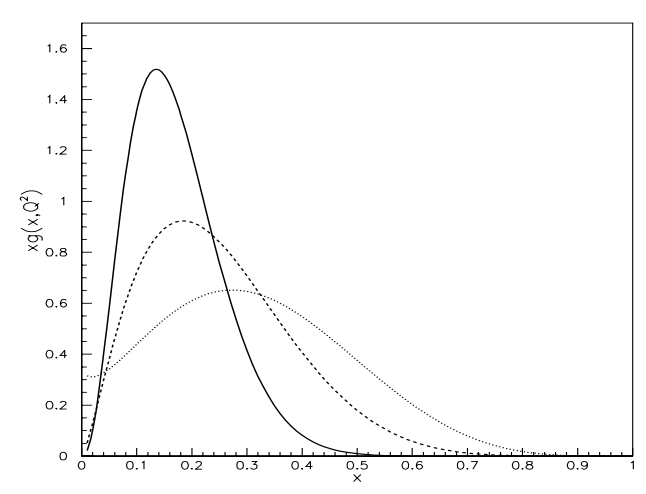

Figure 3: Non-perturbative gluon distribution predicted by the model at $Q_{v}^{2} \simeq 0.64 \mathrm{GeV}^{2}$ (solid line) compared to the valence gluon distribution given by

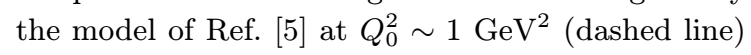
and the initial GRV-94 HO gluon distribution [2] at $Q_{0}^{2}=0.4 \mathrm{GeV}^{2}$ (point line). Model curves were normalized to the value of the integral over $x$ of the GRV-HO gluon distribution.

consequences of making a hadronic Fock state expansion of the nucleon wave-function out of a novel mechanism for generating the cloud. We have shown that within such scheme it is possible to generate not only non-perturbative quarkantiquark distributions but also the gluon sea needed at the low $Q^{2}$ starting scale for DGLAP evolution.

A remarkable feature of the approach is that neutral meson fluctuations are here inhibited and, in turn, non-perturbative gluons take place. The reason is that neutral unflavored structures like the initial $v_{q} \bar{q}$ objects, are more likely to recombine rapidly into a gluon than into a hadron, in contrast to the charged structures like $v_{q} \bar{q}^{\prime}$ which cannot do so. Thus, the hypothetical cloud of virtual fluctuations like $\left|p \pi^{0}\right\rangle$ does not contribute to the sum over $\left|B_{i} M_{i}\right\rangle$ in the RHS of eq. $(1.1)$ but to the second term, $|p g\rangle$, providing a source of valence-like gluons in the proton. It means that, at leading order, only $\left|B \pi^{ \pm}\right\rangle$fluctuations are responsible for the intrinsic sea quark and antiquark distributions at the low $Q^{2}$ scale structure of the nucleon.

Within our model, not only intrinsic quarks and antiquarks but also gluons are generated through quantum fluctuations. When the nucleon fluctuates into a neutral structure plus the nucleon itself, the neutral structure has the quantum numbers of a gluon and is responsible for the non-perturbative, valence-like, gluon sea. In a similar fashion, one can investigate the primordial sea of the pion; see Ref. [ $\left[1 \overline{3}_{1}^{\prime}\right]$ for details.

Concluding, we have proposed a possible scenario for the origin of the valence-like sea quark and gluon distributions nedeed at the low (input) scale in order to describe the experimental DIS data.

\section{Acknowledgments}

H.R.C. would like to acknowledge the Organizing Comitee of the Conference for financial support. J.M. is partially supported by COLCIENCIAS, under Contract No. 242-99.

\section{References}

[1] G. Altarelli and G. Parisi, Nucl. Phys. B126, 298 (1977); Yu. L. Dokshitzer, Sov. Phys. JETP 46 (1977) 641; V.N. Gribov and L.N. Lipatov, Sov.J. Nucl. Phys. 15 (1972) 428.

[2] M. Glück, E. Reya and A. Vogt, Z. Phys. C53, 127 (1992), C67, 433 (1995).

[3] J. Botts et al., Phys. Lett. B304, 159 (1993); H.L. Lai et al., Phys. Rev. D51, 4763 (1995).

[4] A.D. Martin, W.J. Stirling and R.G. Roberts, Phys. Lett. B354, 155 (1995).

[5] A. Edin and G. Ingelman, Phys. Lett. B432, 402 (1998).

[6] S.J. Brodsky, C. Peterson and N. Sakai, Phys. Rev. D23, 2745 (1981).

[7] H.R. Christiansen and J. Magnin, Phys. Lett. B445, 8 (1998).

[8] R.C. Hwa, Phys. Rev. D22, 1593 (1980).

[9] K.P. Das and R.C. Hwa, Phys. Lett B68, 459 (1977).

[10] J. Magnin and H.R. Christiansen, Phys. Rev. D61, 054006 (2000).

[11] E.A. Hawker et al., Phys. Rev. Lett. 80, 3715 (1998); J.C. Peng et al., Phys. Rev. D58, 092004 (1998).

[12] V. Barone, C. Pascaud and F. Zomer, Eur. Phys. J. C12 (2000) 243.

[13] H.R. Christiansen, J. Magnin, hep-ph/0̈003088. 